\title{
Dual mTOR/PI3K inhibitor NVP-BEZ235 arrests colorectal cancer cell growth and displays differential inhibition of 4E-BP1
}

\author{
NAIF ALQURASHI ${ }^{1,2,4}$, SAEED M. HASHIMI ${ }^{1,2}$, FAISALALOWAIDI ${ }^{3}$, SASO IVANOVSKI $^{4}$ and MING Q. WEI $^{4}$ \\ ${ }^{1}$ Department of Basic Sciences, Deanship of Preparatory Studies; \\ ${ }^{2}$ Institute for Research and Medical Consultations, Imam Abdulrahman Bin Faisal University, Dammam 34212; \\ ${ }^{3}$ Department of Pathology and Laboratory Medicine, College of Medicine and University Hospitals, \\ King Saud University, Riyadh 11461, Kingdom of Saudi Arabia; ${ }^{4}$ School of Medical Science \\ and Menzies Health Institute Queensland, Griffith University, Gold Coast, Queensland 4333, Australia
}

Received November 13, 2017; Accepted April 11, 2018

DOI: $10.3892 /$ or.2018.6457

\begin{abstract}
The mammalian target of rapamycin (mTOR), a downstream effector of the PI3K/Akt signalling pathway, is a critical regulator of cell metabolism, growth and survival in response to oncogenic factors. Activation of mTOR frequently occurs in human tumours making it a crucial and validated target in the treatment of cancer. mTOR inhibitors such as rapamycin and its analogues decrease cancer progression in experimental models including colorectal cancer (CRC). Recently, the second generation ATP-competitive mTOR kinase (such as PP242) and dual mTOR/PI3K (such as NVP-BEZ235) inhibitors have entered clinical trials as anticancer agents. However, in CRC, the efficacy of these novel drugs needs to be fully investigated. In the present study, we examined five human CRC cell lines, HT29, HCT116, SW480, SW620 and CSC480 to evaluate their sensitivity to three mTOR inhibitors, RAD001, PP242 and NVP-BEZ235. We observed that compared to RAD001 and PP242, NVP-BEZ235 markedly reduced cell proliferation of CRC cells. Furthermore, we found that the reduced cell proliferation caused by NVP-BEZ235 was not achieved through the disruption of mitochondrial potential. Using an mTOR-specific signalling pathway phospho array we revealed that NVP-BEZ235 significantly decreased phosphorylation of 4E-BP1 (Thr70), the downstream target of mTORC1. In addition, NVP-BEZ235 decreased phosphorylation of AKT (Ser473), the downstream target of mTORC2. Immunoblotting analysis revealed that NVP-BEZ235 effectively inhibited 4E-BP1 phosphorylation, while PP242 had a weak inhibitory
\end{abstract}

Correspondence to: Professor Ming Q. Wei, School of Medical Science and Menzies Health Institute Queensland, Griffith University, Gold Coast, Queensland 4333, Australia

E-mail: m.wei@griffith.edu.au

Key words: mTOR, colorectal cancer, NVP-BEZ235, rapamycin, cell signalling effect. However, PP242 and NVP-BEZ235 decreased AKT levels in all cell lines. RAD001 demonstrated no effect on $4 \mathrm{E}-\mathrm{BP} 1$. Based on the above-mentioned results, the dual $\mathrm{PI} 3 \mathrm{~K} / \mathrm{mTOR}$ and ATP-competitive mTOR inhibitors have demonstrated high potential for targeting the mTOR pathway in CRC.

\section{Introduction}

Colorectal cancer (CRC) is the third most common cancer worldwidewith about 1.4 million new cases diagnosed in 2012 (1). It is predicted that by 2035 the number of diagnosed CRC cases will reach 2.4 million annually worldwide (1). Therefore, developing novel chemotherapeutic agents for the treatment of CRC is an urgent need. Mammalian target of rapamycin (mTOR) is a serine/threonine protein kinase which is a member of the PI3K-related kinase (PIKK) family. In mammalian cells mTOR is composed of two large distinct multi-protein complexes, mTORC1 and mTORC2. mTORC1 consists of mTOR and five accessory proteins whereas mTORC 2 consists of mTOR and six accessory proteins. Both complexes share mTOR as the catalytic subunit of the complex and share mLST8, DEPTOR and Ttil/Tel2 in the complex (2). The unique components of mTORC1 are RAPTOR and PRAS40 whereas RICTOR, mSIN1 and PROTOR are specific to mTORC2 (3). mTORC1 promotes protein synthesis and lipid biogenesis which favours cell growth and proliferation $(3,4)$. mTORC1 plays a central role in tumourigenesis via controlling the mRNA translation and elongation by phosphorylation of its two downstream targets, eukaryotic initiation factor $4 \mathrm{E}$ (eIF4E)-binding protein 1 (4E-BP1) and the p70 ribosomal S6 kinase 1 (S6K1) (2). The resulting activation of S6K1 results in the phosphorylation and inhibition of insulin receptor substrate 1 (IRS1), an upstream regulator of phosphoinositide 3-kinase (PI3K) and AKT kinases. 4E-BP1 regulates the function of eukaryotic translation initiation factor 4E (eIF4E) and when it is phosphorylated by mTORC1 it results in the separation of 4E (eIF4E) from 4E-BP1 and initiates translation by recruiting the translation initiation factor eIF4G to the 5 ' end of 
mRNAs (3). mTORC2 plays a part in tumourigenesis by phosphorylating AKT on the Ser473 residue (5). AKT promotes proliferation, apoptosis and survival through the phosphorylation of several effectors within the mTOR pathway.

Inhibiting mTORC1 and mTORC2 have become attractive therapeutic strategies for cancer including CRC due to their central roles in carcinogenesis. The first-generation mTOR inhibitor rapamycin and its analogues (rapalogs) demonstrated limited efficacy in cancer treatment. These compounds partially suppressed the mTORC1 activity due to incomplete phosphorylation of 4E-BP1 (6). Secondly, they enhanced PI3K/Akt signalling activities via the negative feedback loop that is suppressed upon mTORC1 inhibition resulting in the promotion of cancer cell survival (7). Recently, second generation mTOR inhibitors have emerged including ATP-competitive mTOR kinase and dual mTOR/PI3K inhibitors. They have been used in clinical trials as anticancer agents (8). ATP-competitive mTOR kinase inhibitors (e.g. PP424) target the kinase domain of mTOR and inhibit the kinase activity of both mTORC1 and mTORC2 complexes, there by suppressing the feedback activation of Akt (9). Dual mTOR/PI3K inhibitors (e.g. NVP-BEZ235) inhibit mTORC1, mTORC2 and PI3K by targeting ATP-binding sites of mTOR complexes and the $\mathrm{p} 110 \alpha, \beta$, and $\gamma$ isoforms of PI3K leading to complete blockage of the PI3K/Akt pathway (10).

A growing body of evidence indicates that mTORC1 and mTORC2 are overexpressed in human CRCs and the inhibition of these components inhibits cell proliferation and induces apoptosis in in vitro and in vivo models $(11,12)$. The aim of thepresent study was to compare the antitumor activity of ATP competitive mTOR kinase inhibitor PP424 with dual $\mathrm{PI} 3 \mathrm{~K} / \mathrm{mTOR}$ inhibitor NVP-BEZ235 in human CRC cell lines. Our findings provided evidence supporting the notion that targeting mTOR pathway with PP424 and NVP-BEZ235 could be useful as a potential therapeutic strategy for anticancer therapy in CRC.

\section{Materials and methods}

Cell culture and reagents. NVP-BEZ235 and PP242 were purchased from ChemdeaLLC (Ridgewood, NJ, USA) and used at a concentration of $1,000 \mathrm{nM}$ in all experiments. Rapamycin (RAD001) was obtained from LC Laboratories (Woburn, MA, USA) and used at a concentration of 1,000 nM. Each inhibitor was dissolved in dimethyl sulfoxide (DMSO) and stored at $-20^{\circ} \mathrm{C}$ until use. All drugs were diluted in culture medium immediately before use. The human CRC cell lines HT29, HCT116, SW620 and SW480 were purchased from the American Type Culture Collection (ATCC; Manassas, VA, USA). CSC480 cell line was purchased from BioMedicure (San Diego, CA, USA) (13). All cell lines were cultured in Dulbecco's modified Eagle's medium (DMEM), $4.5 \mathrm{~g} / 1$ D-glucose, L-glutamine and $110 \mathrm{mg} / \mathrm{l}$ sodium pyruvate (all from Invitrogen; Thermo Fisher Scientific, Inc., Waltham, MA, USA) containing $10 \%$ fetal bovine serum (FBS; Invitrogen; Thermo Fisher Scientific, Inc.) at $37^{\circ} \mathrm{C}$ in a humidified atmosphere consisting of $5 \% \mathrm{CO}_{2}$ and $95 \%$ air. Cells were starved $24 \mathrm{~h}$ in serum-free medium and stimulated with insulin $(200 \mathrm{nM})$ for $10 \mathrm{~min}$. All primary antibodies against, pan 4E-BP1 (1:1,000; cat. no. 9644), pho-4E-BP1 (Thr37/46)
(1:1,000; cat. no. 9459), pan AKT (1:1,000; cat. no. 4691) and pho-AKT (Ser473) (1:2,000; cat. no. 4060) were purchased and validated by the manufacturer (Cell Signalling Technology, Inc., Danvers, MA, USA).

Cell viability analysis. Determination of cell viability was performed by the 3-[4,5-dimethylthiazol-2-yl]-2,5-diphenyl-tetrazolium bromide (MTT) assay under both normoxic and hypoxic conditions. CRC cells were seeded into 96-well plates in triplicate at a density of $1 \times 10^{4}$ cells/well. After $24 \mathrm{~h}$, the cells were exposed to different concentrations of RAD001, PP242 and NVP-BEZ235 $(0.1-10,000 \mathrm{nmol} / \mathrm{l})$ in $100 \mu 1$ of medium. After incubation for the indicated time-points, the MTT reagent (Life Technologies; Thermo Fisher Scientific, Inc.) was added to each well, following the manufacturer's instructions and incubated (at $37^{\circ} \mathrm{C}$ with $5 \% \mathrm{CO}_{2}$ ) for another $2 \mathrm{~h}$ and the medium was removed. For the hypoxic treatment, the cells were incubated in an anaerobic chamber (Forma Anaerobic System; Thermo Fisher Scientific, Inc.). DMSO (100 $\mu \mathrm{l})$ was added to each well to dissolve the dark purple crystal formed, and the plate was shaken gently for $5 \mathrm{~min}$ at room temperature. Subsequently, absorbance was assessed at $540 \mathrm{~nm}$ using an absorbance plate reader (POLARstar Omega reader; BMG Labtech, Cary, NC, USA). For examining the effect of removing NVP-BEZ235, after three days the cells were cultured with drug-free DMEM for another three days.

Cell proliferation assay. The cells were seeded in 100-mm plates at $1 \times 10^{6}$ cells in $10 \mathrm{ml}$ of media. After $24 \mathrm{~h}$, cells were treated with RAD001, PP242 and NVP-BEZ235 $(1 \mu \mathrm{M})$. After incubation at $37^{\circ} \mathrm{C}$ with the drugs for $1,2,3,4$ or 5 days, the cells were extensively rinsed in phosphate-buffered saline (PBS) to remove any loosely attached or floating cells. The untreated control and treated cells were harvested by trypsinization and cell numbers were determined using a hemocytometer.

Mitochondrial membrane potential assay. Mitochondrial membrane potential was determined by staining cells with 5,5',6,6'-tetrachloro-1,1',3,3'-tetra-ethylbenzimidazole carbocyanide iodide (JC-1) fluorescence dye (Sigma-Aldrich; Merck KGaA, Darmstadt, Germany). JC-1 fluorescence emanating from the mitochondria was analysed by fluorescence microscopy (14). Cells were incubated with JC-1 $(2.5 \mu \mathrm{mol} / \mathrm{l})$ in 96-well plates at $37^{\circ} \mathrm{C}$ and $5 \% \mathrm{CO}_{2}$ for $30 \mathrm{~min}$. Drugs $(1 \mu \mathrm{mol} / \mathrm{l})$ and valinomycin $(1 \mu \mathrm{mol} / \mathrm{l})$ were added $30 \mathrm{~min}$ before JC-1. Red fluorescence (excitation $550 \mathrm{~nm}$, emission $600 \mathrm{~nm}$ ) and green fluorescence intensity (excitation $485 \mathrm{~nm}$ and emission $535 \mathrm{~nm}$ ) were detected using a POLARstar Omega microplate reader (BMG Labtech).

Phospho-specific protein microarray analysis. The mTORsignalling phospho-specific antibody array was conducted (FullMoon BioSystems, Inc., Sunnyvale, CA, USA) according to the mancufacturer's protocol. The array consisted of 138 phospho-specific antibodies against phosphorylated and unphosphorylated proteins. All antibodies were related to proteins involved in the mTOR pathway. Briefly, SW620 cells were serum-starved for $24 \mathrm{~h}$ and treated with vehicle or NVP-BEZ235 $(10 \mu \mathrm{M})$ for $2 \mathrm{~h}$. Untreated cells served as controls. Cells were lysed using protein extraction buffer 
Table I. $\mathrm{IC}_{50}$ values obtained after the CRC cell lines were treated with mTOR inhibitors.

\begin{tabular}{lrrrrrrr}
\hline & \multicolumn{3}{c}{ Normoxia } & & \multicolumn{3}{c}{ Hypoxia } \\
\cline { 2 - 3 } Cell line & RAD001 & PP242 & NVP-BEZ235 & & RAD001 & PP242 & NVP-BEZ235 \\
\hline HT29 & 7731.60 & 445.13 & 50.14 & & 10699.68 & 4598.27 & 480.36 \\
HCT116 & 8640.46 & 1966.92 & 27.58 & & $<>$ & 4.40 & 3.25 \\
SW620 & 523.39 & 62.24 & 0.50 & & 11696.43 & 2711.80 & 58.35 \\
SW480 & 3484.61 & 169.11 & 0.47 & & 860.41 & 3932.78 & 81.00 \\
CSC480 & 3983.25 & 6500.46 & 281.63 & & 11769.11 & 9548.71 & $\diamond$ \\
\hline
\end{tabular}

CRC, colorectal cancer; $<>$ indicates that $\mathrm{IC}_{50}$ could not be calculated.

provided by Full Moon BioSystems Inc. Proteins were labelled using biotin dissolved in N,N-dimethylformamide (DMF) and hybridised to the (76x25 mm) microarray slides. Biotin-labelled proteins conjugated to antibodies were detected using streptavidin-Cy3 (Full Moon BioSystems). Slides were scanned using GenePix 4100 microarray scanner (Molecular Devices, San Jose, CA, USA). Phosphorylated protein expression was normalized to its corresponding total protein. Fold change in intensities from six replicates between the phosphorylation of SW620-treated and untreated cells was calculated. Ratios of phosphorylated to unphosphorylated proteins were calculated as follows: Average signal intensity of phospho-site-specific antibody/average signal intensity of site-specific antibody. The ratio change between samples was calculated as follows: Treated/control.

Protein extraction and immunoblot analysis. HT29, HCT116, SW620, SW480 and CSC480 cells were serum starved for $24 \mathrm{~h}$. Subsequently, the cells were pre-treated with drugs $(1 \mu \mathrm{mol} / \mathrm{l})$ or DMSO as a control for $2 \mathrm{~h}$ in the presence of insulin $(200 \mathrm{nmol} / \mathrm{l})$. For protein lysates, cells were washed extensively with ice-cold PBS. Cells were subsequently harvested in RIPA lysis buffer containing $50 \mathrm{mM}$ Tris- $\mathrm{HCl}$, pH 7.4, $150 \mathrm{mM} \mathrm{NaCl}, 1 \%$ Nonidet P-40, $0.5 \%$ sodium deoxycholate, $1 \%$ SDS, $5 \mathrm{mM}$ EDTA, $1 \mathrm{mM}$ phenylmethylsulfonyl fluoride, $10 \mu \mathrm{g} / \mathrm{ml}$ leupeptine and $1 \mathrm{mM}$ sodium orthovanadate. RIPA lysis buffer was supplemented with Protease and Phosphatase Inhibitor Cocktail (cat. no. 78440; Thermo Fisher Scientific, Inc.). Lysates were used immediately or stored at $-80^{\circ} \mathrm{C}$ for later use. Protein concentrations were assessed using POLARstar Omega (BMG Labtech). For immunoblotting, equal amounts of lysates $(20 \mu \mathrm{g})$ were diluted in reducing 4XSDS buffer, boiled and then separated on 4-15\% polyacrylamide gel (Mini-PROTEAN TGX; Bio-Rad Laboratories, Inc., Hercules, CA, USA). Subsequently, the resolved proteins were transferred onto polyvinylidene difluoride (PVDF) membrane (Trans-Blot Turbo Transfer $0.2 \mu \mathrm{m}$ PVDF; Bio-Rad Laboratories). Membranes were blocked with Tris buffered saline (TBS) in 1\% BSA pH 8.0 (Sigma-Aldrich). Membranes were washed and incubated with primary antibodies overnight at $4^{\circ} \mathrm{C}$. After extensive washing with TBS plus $0.1 \%$ Tween-20 (TBST), the membranes were incubated with appropriate anti-rabbit IgG, HRP-linked secondary antibody (1:1,000; cat. no. 7074) (Cell Signaling Technology, Inc.). Following 1-h incubation, the membranes were washed with TBST and the proteins of interest were detected by using Super Signal West Pico substrate (Thermo Fisher Scientific, Inc.). Bands from immunoreactive proteins were visualised by VersaDoc imaging system (Bio-Rad Laboratories).

Statistical analysis. Curve fitting was conducted using GraphPad Prism ver. 6 (GraphPad Software, Inc., La Jolla, CA, USA). Data are presented as the mean of at least three replicates with standard error of the mean. Student's t-test was used to analyse the data and a significance level of $\mathrm{P}<0.05$ was used to indicate statistically significant differences. In the Student's t-test each drug group was individually compared to the control group (no drug) and the multiple group graphs represented significance in relation to the no drug control.

\section{Results}

mTOR inhibitors suppress CRC cell proliferation in vitro. We evaluated the effects of mTOR inhibitors on CRC cells in vitro using the cell viability and proliferation assays under both normoxic and hypoxic conditions. To explore the effect of mTOR inhibitors on CRC cells, serial dilutions of RAD001, PP242 and NVP-BEZ235 ranging from 0.1 to $10,000 \mathrm{nM}$ were added to different CRC cell lines, including HT29, HCT116, SW620 and SW480, as well as CSC480 cells. For MTT assay, a comparison was made among RAD001, PP242 and NVP-BEZ235 in normoxia and hypoxia. $\mathrm{IC}_{50}$ values were evaluated using the MTT assay. Table I displays the $\mathrm{IC}_{50}$ values obtained after the cell lines were treated with the mTOR inhibitors. In normoxia, a progressive decrease of the viable cell numbers was observed on different CRC cell lines by treating the cells with increasing concentrations of the drugs (Fig. 1A). The capacity of RAD001 and PP242 to reduce cell proliferation was limited, except for PP242 having an increased inhibitory effect on SW620 cells $\left(\mathrm{IC}_{50}, 62 \mathrm{nmol} / \mathrm{l}\right)$. Most cell lines displayed an $\mathrm{IC}_{50}$ for NVP-BEZ235 ranging from 0.47 to $50 \mathrm{nmol} / 1$, whereas the $\mathrm{IC}_{50}$ for CSC480 cells was $282 \mathrm{nmol} / \mathrm{l}$. The CRC cell lines SW480 and SW620 had the highest sensitivity to NVP-BEZ235 $\left(\mathrm{IC}_{50}, 0.47\right.$ and $0.5 \mathrm{nmol} / \mathrm{l}$, respectively). In hypoxia, the cell lines demonstrated more resistance to the drugs except for HCT116 cells. PP242 
A
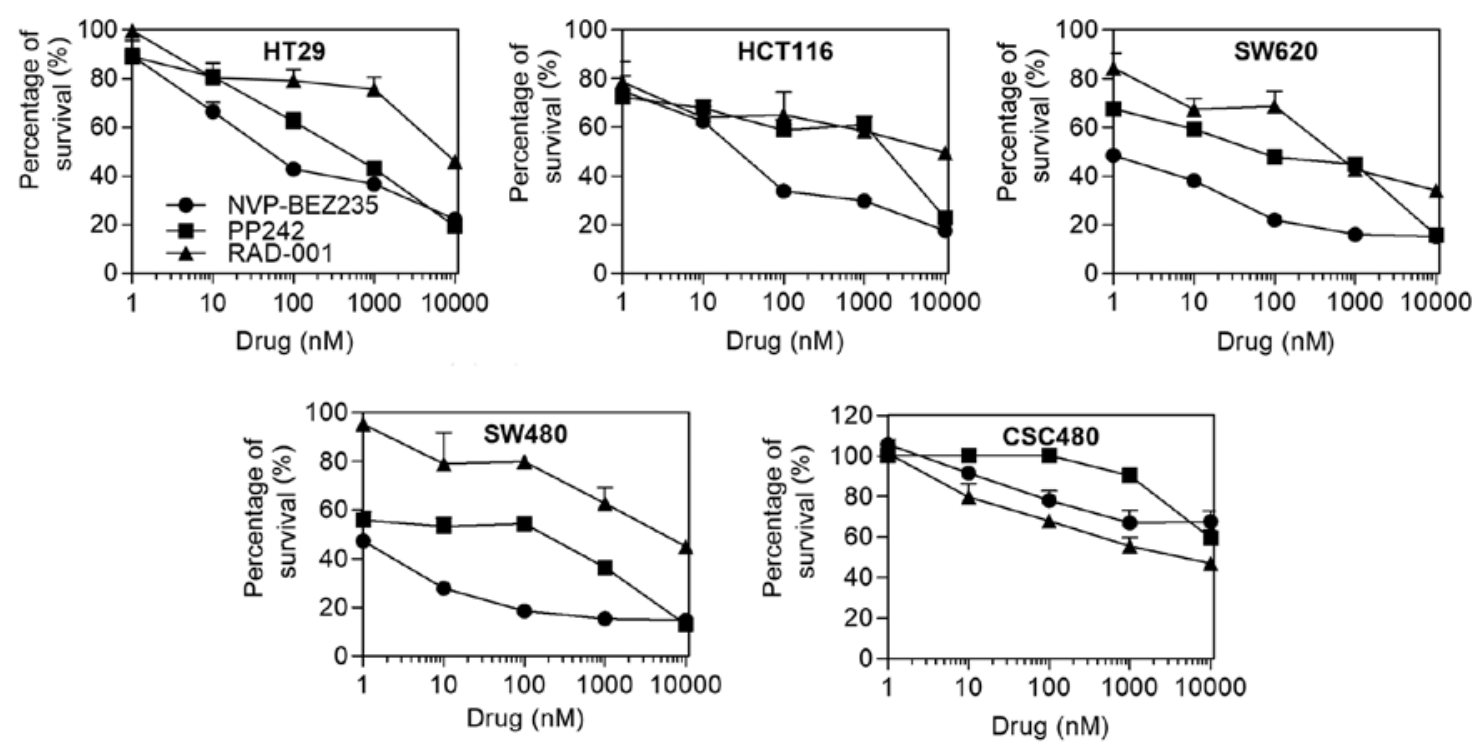

B
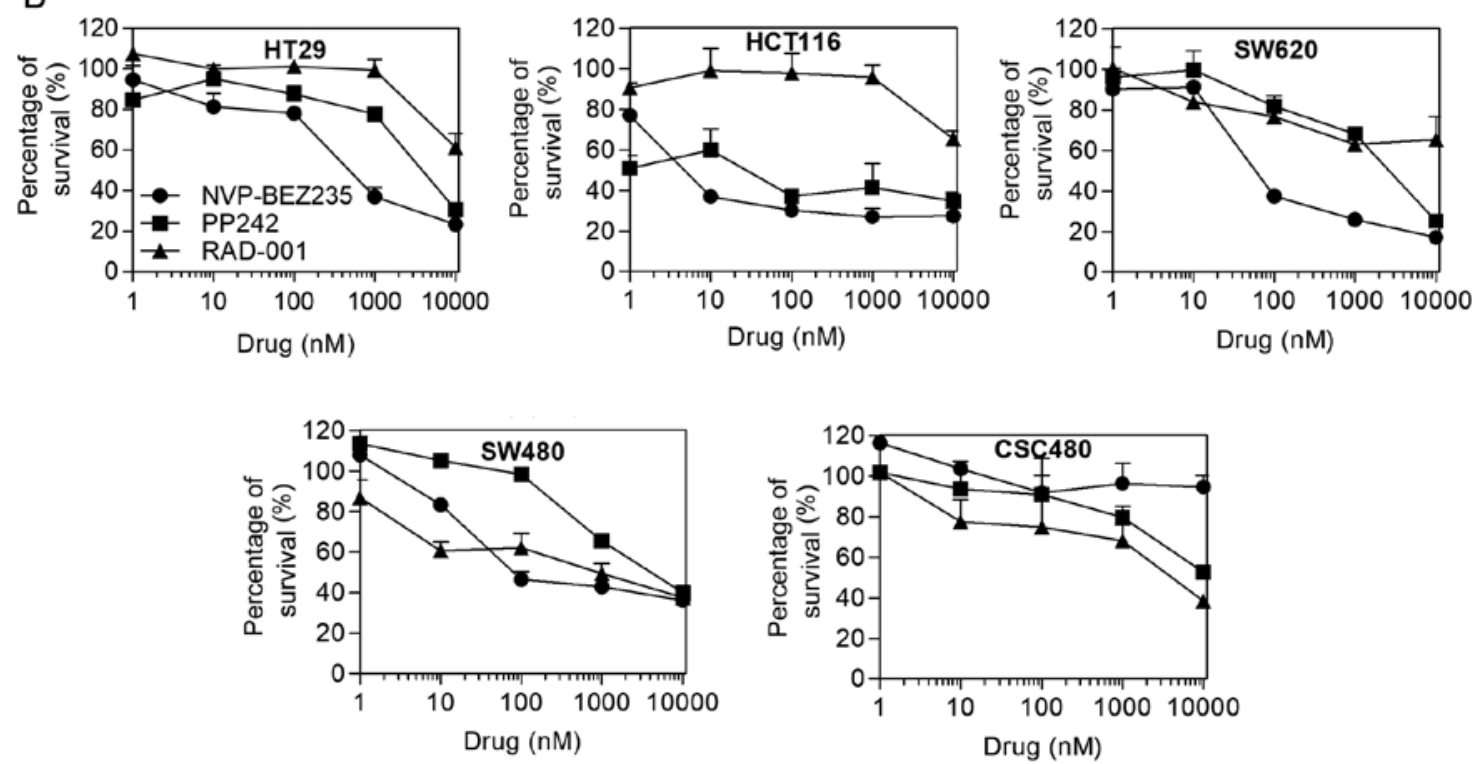

Figure 1. Cell viability of HT29, HCT116, SW620, SW480 and CSC480 cells under normoxic and hypoxic conditions. (A) CRC cell lines were assessed by an MTT assay after treatment with increasing concentrations (0.1-10,000 nM) of RAD001, PP242 and NVP-BEZ235 for 48 h. RAD001 demonstrated limited efficacy in vitro. PP242 enhanced cytotoxic effect at relatively high concentrations in HT29, HCT116, SW620 and SW480 cells. NVP-BEZ235 treatment resulted in significantly decreased cell viability. (B) Cells were exposed to $0.5 \% \mathrm{O}_{2}$ for $24 \mathrm{~h}$, and then treated with increasing concentrations (0.1-10,000 $\mathrm{nM}$ ) of drugs for $24 \mathrm{~h}$. The cell viability was assessed by the MTT assay. PP242 and NVP-BEZ235 had strong effects on the viability of HCT116 cells.

$\left(\mathrm{IC}_{50}, 4.4 \mathrm{nmol} / \mathrm{l}\right)$ and NVP-BEZ235 ( $\left.\mathrm{IC}_{50}, 3.25 \mathrm{nmol} / \mathrm{l}\right)$ had significant effects on HCT116 cell viability (Fig. 1B). Notably, in both normoxic and hypoxic conditions, RAD001 exhibited better effect than NVP-BEZ235 in SW480 and CSC480.

Subsequently, we evaluated the capacity of mTOR inhibitors to reduce cell growth at different time-points by proliferation assay. Using these cell lines, in vitro growth-inhibitory effect of mTOR inhibitors was examined in $1 \mu \mathrm{M}$ concentration of the drugs. Although some cell lines displayed sensitivity to RAD001 and PP242 treatment at days 2 and 3, the cells started to proliferate again thereafter, resulting in a 2 to 3 -fold increase in the number of cells (Fig. 2). In contrast, when all cell lines were treated by NVP-BEZ235, it caused persistent inhibition of cell growth, which remained at a low level thereafter.
Mitochondrial membrane potentials. To examine whether targeting mTOR induced collapse of the mitochondrial membrane potential $(\Delta \Psi \mathrm{m})$, we used JC-1 dye, which concentrates in the mitochondrial matrix of healthy cells forming red fluorescent aggregates at high-membrane potential. In apoptotic cells, the dye is dispersed throughout the entire cell leading to a shift from red to green fluorescence. RAD001 and PP242 slightly induced mitochondrial dysfunction and activated cell apoptosis in SW620 cells (Fig. 3). Notably, in the present study, there was no significant difference among other mTOR inhibitor-treated groups compared to the control groups (Fig. 3). Valinomycin was used as a positive control which collapsed $\Delta \Psi \mathrm{m}$ and indicated cell apoptosis or necrosis. 

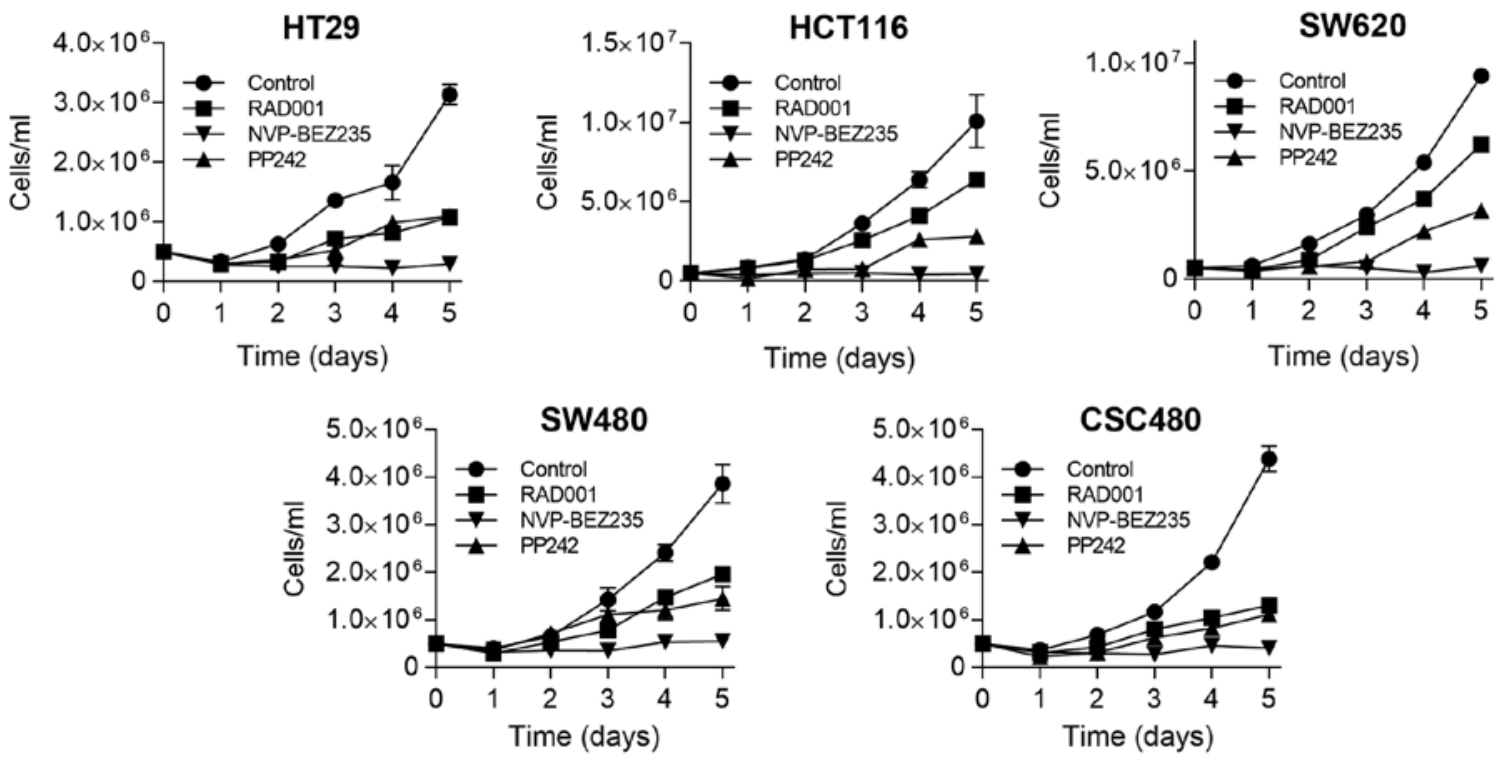

Figure 2. Proliferation assays. Antiproliferative effect on HT29, HCT116, SW620, SW480 and CSC480 cells at different time-points after treatment with $1 \mu \mathrm{M}$ of RAD001, PP242 and NVP-BEZ235. When compared with control/vehicle NVP-BEZ235 inhibited cell proliferation in all cell lines, while RAD001 demonstrated a cytotoxic effect at day 1,2 and 3 in HT29, SW480 and CSC480, whereas HCT116 and SW620 were affected on day 2.
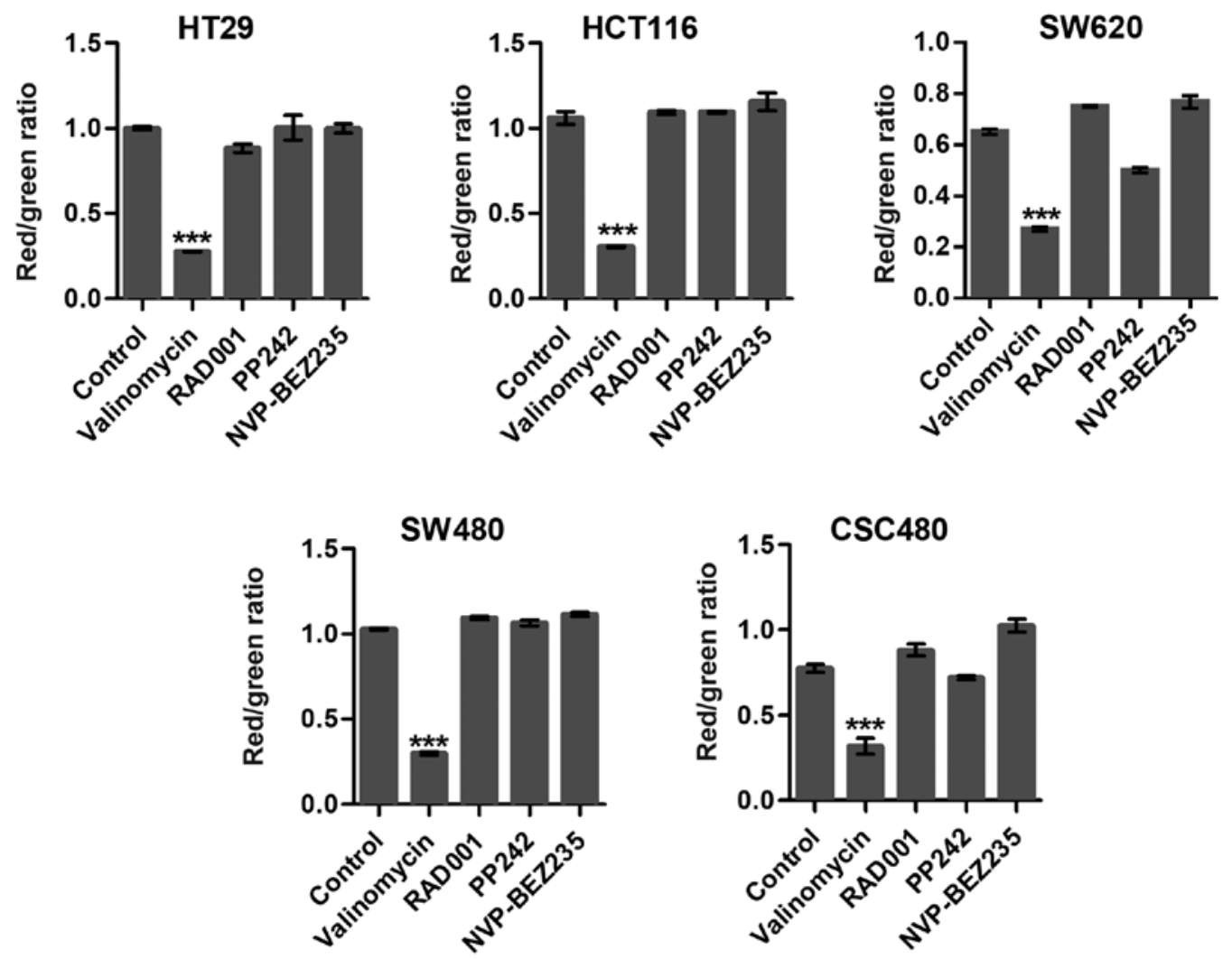

Figure 3. JC-1 staining for mitochondrial potential $(\Delta \Psi \mathrm{m})$ after mTOR inhibitor treatment. Cells were treated with mTOR inhibitors at $1 \mu \mathrm{M}$ for $24 \mathrm{~h}$; $1 \times 10^{4}$ cells in 96-well plate (4-wells for each group) were incubated in $100 \mu \mathrm{l}$ culture medium and loaded with mitochondrial potential sensor JC-1 (10 $\left.\mu \mathrm{g} / \mathrm{ml}\right)$ for $10 \mathrm{~min}$ at $37^{\circ} \mathrm{C}$. Valinomycin is a known disruptor of mitochondrial potential and was used as a positive control. ${ }^{* * *}$ Indicates $\mathrm{P}<0.001$.

mTOR phospho-antibody microarray reveals effect of mTOR inhibitors. To achieve a comprehensive understanding of the behaviour of key proteins within the mTOR pathway under inhibitory conditions, SW620 cells were treated with the dual specificity inhibitor NVP-BEZ235 and the phosphorylation status of the proteins was analysed using anmTOR specific antibody phospho array. The array contained 138 highly specific and well-characterized phospho-specific antibodies for upstream and downstream proteins in the mTOR pathway. To allow determination of the relative level of 
Table II. Fold change in phosphorylated proteins in response to drugs.

\begin{tabular}{|c|c|c|c|c|}
\hline \multirow[b]{2}{*}{ Phosphorylation site } & \multirow[b]{2}{*}{ Swiss-Prot No. } & SW620 Non & SW620 NVP & $\begin{array}{l}\text { SW620 NVP/ } \\
\text { SW620 Non }\end{array}$ \\
\hline & & \multicolumn{3}{|c|}{ Fold change } \\
\hline GSK3 beta (Phospho-Ser9) & P49841 & 1.65 & 3.50 & 2.12 \\
\hline PIP5K (Phospho-Ser307) & Q9Y2I7 & 0.68 & 1.14 & 1.68 \\
\hline $\begin{array}{l}\text { 6-Phosphofructo-2-kinase/fructose-2,6-biphosphatase } 2 \\
\text { (PFKFB2) (Phospho-Ser483) }\end{array}$ & O60825 & 0.81 & 1.36 & 1.68 \\
\hline PTEN (Phospho-Ser370) & P60484 & 1.76 & 2.85 & 1.61 \\
\hline P90RSK (Phospho-Thr573) & Q15418 & 0.13 & 0.17 & 1.38 \\
\hline eIF4G (Phospho-Ser1108) & Q04637 & 0.46 & 0.60 & 1.31 \\
\hline PTEN (Phospho-Ser380/Thr382/Thr383) & P60484 & 2.75 & 3.57 & 1.30 \\
\hline BAD (Phospho-Ser136) & Q92934 & 1.82 & 2.33 & 1.28 \\
\hline RSK1/2/3/4 (Phospho-Ser221/227/218/232) & $\begin{array}{l}\text { Q15418/ } \\
\text { P51812/ } \\
\text { Q15349/ } \\
\text { Q9UK32 }\end{array}$ & 1.38 & 1.69 & 1.22 \\
\hline P70S6K (Phospho-Thr229) & P23443 & 0.85 & 1.00 & 1.18 \\
\hline mTOR (Phospho-Ser2448) & $\mathrm{P} 42345$ & 1.02 & 1.17 & 1.16 \\
\hline AKT1 (Phospho-Thr450) & P31749 & 0.87 & 0.95 & 1.09 \\
\hline P90RSK (Phospho-Thr359/Ser363) & Q15418 & 0.18 & 0.19 & 1.03 \\
\hline P70S6K (Phospho-Thr421) & P23443 & 1.46 & 1.48 & 1.01 \\
\hline AKT1S1 (Phospho-Thr246) & Q96B36 & 0.30 & 0.30 & 1.00 \\
\hline BAD (Phospho-Ser91/128) & Q92934 & 5.67 & 5.12 & 0.90 \\
\hline AMPKbeta1 (Phospho-Ser182) & Q9Y478 & 0.85 & 0.76 & 0.89 \\
\hline 4E-BP1 (Phospho-Ser65) & Q13541 & 0.34 & 0.30 & 0.89 \\
\hline PTEN (Phospho-Ser380) & P60484 & 1.10 & 0.95 & 0.86 \\
\hline PDK1 (Phospho-Ser241) & O15530 & 0.76 & 0.62 & 0.82 \\
\hline AMPK1/AMPK2 (Phospho-Ser485/491) & $\begin{array}{l}\text { Q13131/ } \\
\text { P54646 }\end{array}$ & 3.25 & 2.59 & 0.80 \\
\hline BAD (Phospho-Ser134) & Q92934 & 0.72 & 0.57 & 0.79 \\
\hline PKC alpha/beta II (Phospho-Thr638) & P17252 & 1.30 & 0.95 & 0.73 \\
\hline P70S6k-beta (Phospho-Ser423) & Q9UBS0 & 6.21 & 4.32 & 0.70 \\
\hline $\begin{array}{l}\text { PI3-kinase p85-subunit alpha/gamma } \\
\text { (Phospho-Tyr467/Tyr199) }\end{array}$ & $\begin{array}{l}\text { P27986/ } \\
\text { Q92569 }\end{array}$ & 2.05 & 1.40 & 0.68 \\
\hline AKT1 (Phospho-Ser246) & P31749 & 1.54 & 1.05 & 0.68 \\
\hline AKT (Phospho-Thr308) & P31749 & 2.04 & 1.37 & 0.67 \\
\hline AKT1 (Phospho-Ser124) & P31749 & 0.83 & 0.53 & 0.64 \\
\hline PPAR-b (Phospho-Thr1457) & Q15648 & 1.52 & 0.96 & 0.63 \\
\hline Mnk1 (Phospho-Thr385) & Q9BUB5 & 1.57 & 0.95 & 0.60 \\
\hline AKT (Phospho-Ser473) & P31749 & 2.11 & 1.27 & 0.60 \\
\hline $\begin{array}{l}\text { Rho/Rac guanine nucleotide exchange factor } 2 \\
\text { (Phospho-Ser885) }\end{array}$ & Q8TDA3 & 2.14 & 1.28 & 0.60 \\
\hline ERK3 (Phospho-Ser189) & Q16659 & 3.00 & 1.77 & 0.59 \\
\hline BAD (Phospho-Ser112) & Q92934 & 2.31 & 1.33 & 0.58 \\
\hline GSK3 alpha (Phospho-Ser21) & P49840 & 2.79 & 1.56 & 0.56 \\
\hline PKC alpha (Phospho-Tyr657) & P17252 & 4.13 & 2.29 & 0.55 \\
\hline GSK3a-b (Phospho-Tyr216/279) & P49840 & 4.49 & 2.49 & 0.55 \\
\hline AKT2 (Phospho-Ser474) & P31751 & 2.63 & 1.44 & 0.55 \\
\hline mTOR (Phospho-Thr2446) & P42345 & 4.08 & 2.21 & 0.54 \\
\hline PPAR-r (Phospho-Ser112) & P37231 & 0.16 & 0.08 & 0.51 \\
\hline AKT1 (Phospho-Tyr474) & P31749 & 0.64 & 0.32 & 0.51 \\
\hline eIF2 alpha (Phospho-Ser51) & P05198 & 2.33 & 1.14 & 0.49 \\
\hline
\end{tabular}


Table II. Continued.

\begin{tabular}{|c|c|c|c|c|}
\hline \multirow[b]{2}{*}{ Phosphorylation site } & \multirow[b]{2}{*}{ Swiss-Prot No. } & SW620 Non & SW620 NVP & $\begin{array}{l}\text { SW620 NVP/ } \\
\text { SW620 Non }\end{array}$ \\
\hline & & \multicolumn{3}{|c|}{ Fold change } \\
\hline PP2A-a (Phospho-Tyr307) & P67775 & 0.39 & 0.18 & 0.47 \\
\hline eIF4E (Phospho-Ser209) & P06730 & 2.10 & 0.96 & 0.46 \\
\hline AKT1 (Phospho-Thr72) & P31749 & 0.34 & 0.15 & 0.45 \\
\hline P70S6K (Phospho-Ser418) & P23443 & 0.95 & 0.42 & 0.44 \\
\hline P70S6K (Phospho-Ser411) & P23443 & 2.90 & 1.18 & 0.41 \\
\hline BAD (Phospho-Ser155) & Q92934 & 3.39 & 1.34 & 0.39 \\
\hline AKT (Phospho-Tyr326) & P31749 & 1.73 & 0.64 & 0.37 \\
\hline P70S6K (Phospho-Ser371) & P23443 & 1.68 & 0.61 & 0.37 \\
\hline mTOR (Phospho-Ser2481) & P42345 & 2.17 & 0.80 & 0.37 \\
\hline 4E-BP1 (Phospho-Thr70) & Q13541 & 2.55 & 0.91 & 0.36 \\
\hline Tuberin/TSC2 (Phospho-Thr1462) & P49815 & 0.50 & 0.17 & 0.35 \\
\hline AMPK1 (Phospho-Thr174) & Q13131 & 2.57 & 0.88 & 0.34 \\
\hline P90RSK (Phospho-Ser380) & Q15418 & 0.94 & 0.32 & 0.34 \\
\hline ERK1-p44/42 MAP Kinase (Phospho-Tyr204) & $\begin{array}{l}\text { P27361/ } \\
\text { P28482 }\end{array}$ & 5.45 & 1.84 & 0.34 \\
\hline ERK1-p44/42 MAP Kinase (Phospho-Thr202) & $\begin{array}{l}\text { P27361/ } \\
\text { P28482 }\end{array}$ & 5.98 & 1.92 & 0.32 \\
\hline Tuberin/TSC2 (Phospho-Ser939) & P49815 & 0.64 & 0.18 & 0.28 \\
\hline P70S6K (Phospho-Ser424) & P23443 & 5.96 & 1.36 & 0.23 \\
\hline
\end{tabular}

SW620 Non, non-treated cells; SW620 NVP, cells treated with NVP-BEZ235.

phosphorylation, pairs of antibodies (phosphorylated and unphosphorylated) for the same target sites were included in the array. The antibody array data revealed that NVP-BEZ235 decreased phosphorylation of AKT on Ser473 by 0.6 -fold. In addition, phosphorylation of AKT on the other site Thr308 decreased by 0.7 -fold. Furthermore, the data demonstrateddifferential regulation of many proteins related to the mTOR pathway (Table II). For example, phosphorylation of mTOR at Ser2481 was decreased by about 0.4 -fold. In response to NVP-BEZ235 treatment, a negative regulator of mTORC1, TSC2 protein tuberin decreased by 0.35 -fold at Thr1462 and 3 -fold at Ser939.

PP242 and NVP-BEZ235 treatment of human colorectal cell lines results in sustained mTORC1 and mTORC2 inhibition. To examine the effects of RAD001, PP242 and NVP-BEZ235 treatment on mTORC1 (p4E-BP1 T37/46) and mTORC2 (pAKT S473) mTOR signalling, western blot analysis was performed. After 2-h treatment of HT29, HCT116, SW620, SW480 and CSC480 cells with all agents at concentrations of $1 \mu \mathrm{mol} / 1$ (Fig. 4), the expression of non-phosphorylated AKT and 4E-BP1 (functionally inactive) was unchanged in all cell lines. However, the expression of phosphorylated AKT (pAKT, functionally active) and phosphorylated p4E-BP1 (p4E-BP1, functionally active) (Fig. 4) was differentiated in response to different drugs. Concerning pAKT S473, PP242 and NVP-BEZ235 were able to inhibit phosphorylation of S473 in all cell lines (Fig. 4). In the same cell lines, the phosphorylation status of pAKT S473 was not affected by RAD001. In contrast, the expression of p4E-BP1 T37/46 was not affected by PP242 in three cell lines (SW620, SW480 and CSC480). However, the expression decreased in HCT116 cells and showed full inhibition in HT29 cells in response to PP242 treatment. NVP-BEZ235 inhibited phosphorylation of T37/46 in HCT116, HT29, SW620 and CSC480. Notably, NVP-BEZ235 had no effect on the expression of p4E-BP1 (T37/46) in SW480 cells. Collectivelly, these data indicated that treatment by PP242 and NVP-BEZ235 resulted in sustained inhibition of mTORC1 and mTORC2 signalling in vitro.

\section{Discussion}

Rapamycin and its analogues are inhibitors for the mTOR signalling pathway. Previous research revealed that they have antitumour activities through binding to its intracellular receptor FKBP12 forming a rapamycin-FKBP12 complex which binds directly to mTORC1 and indirectly to mTORC2 inhibiting their activities (15). Other studies also indicated that inhibiting the S6K1-IRS1 feedback loop by rapamycin leads mTORC1 to hyper-activate the PI3K-Akt pathway (16). However, treatment of cancer with rapamycin has limited efficacy in the mTOR pathway (17). Recent advances in the development of next generation mTOR inhibitors PP242 (ATP-competitive mTOR 

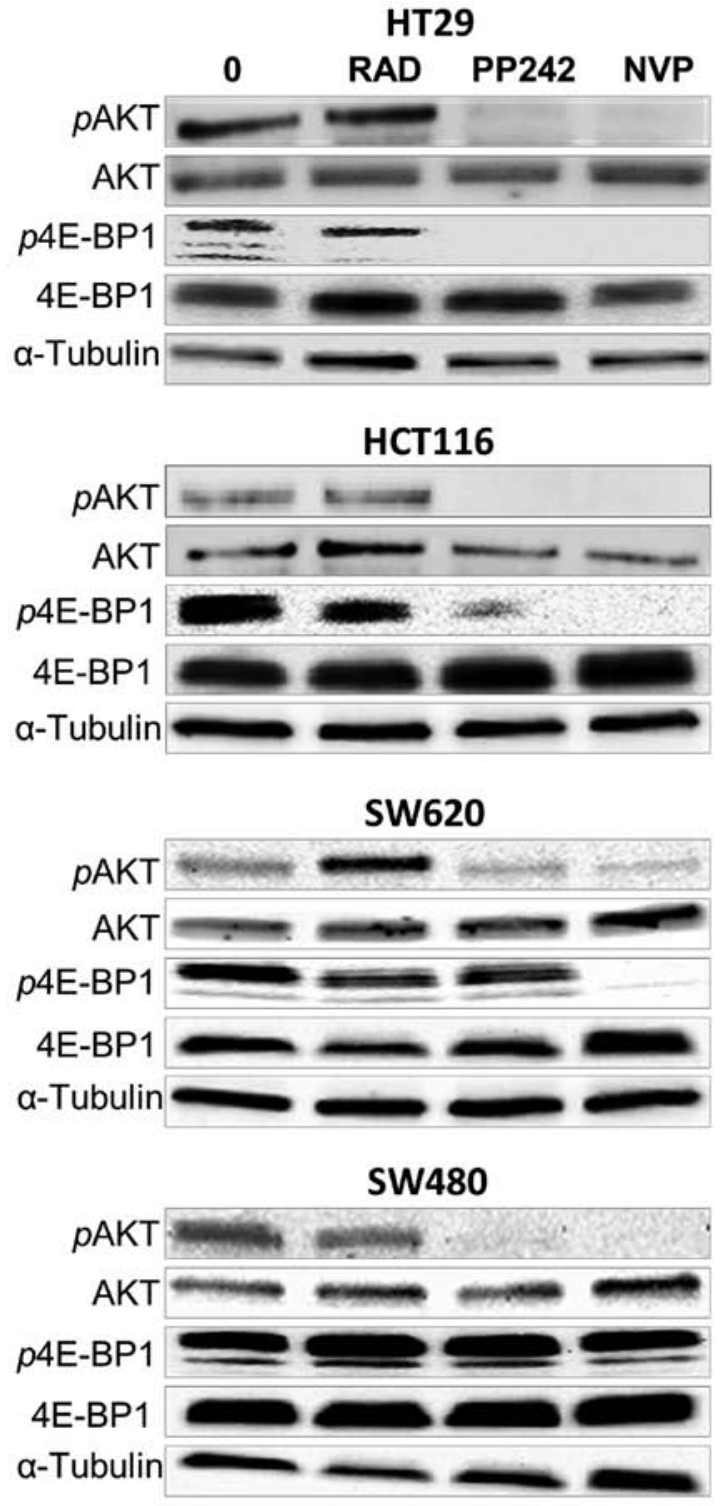

\section{$\operatorname{csc480}$}

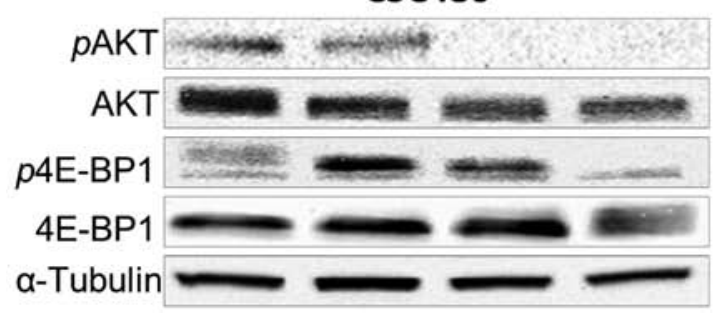

Figure 4. Western blot analysis of key mTOR signalling proteins. Immunoblotting was performed on pAKT (S473), AKT, p4E-BP1 (T37/46), 4E-BP1 and $\alpha$-tubulin in cells treated with RAD001, PP242 and NVP-BEZ235. HT29, HCT116, SW620, SW480 and CSC480 cells were treated with vehicle $(0.1 \%$ DMSO) or drugs $(1.0 \mu \mathrm{M})$ for $2 \mathrm{~h}$.

inhibitor) and NVP-BEZ235 (dual PI3K-mTOR inhibitor) have the potential to overcome the development of drug resistance due to targeting of the mTOR pathway at multiple levels (18). PP242 and NVP-BEZ235 target both mTOR complexes and upstream activators and downstream targets of mTOR, providing promising approaches for cancer therapy $(10,19)$.

In the present study, we used multiple human CRC cell lines (HT29, HCT116, SW620, SW480 and CSC480) to evaluate the interaction of mTORC1 and mTORC2 with their substrate targets 4E-BP1 and AKT, respectively. Furthermore, we investigated the role of mTORC1 and mTORC2 in human CRC using the new generation of mTOR inhibitors, PP242 and NVP-BEZ235.

In cancer cells, mTOR signalling induces cell proliferation in two ways, downstream of mTORC1 at the level of 4E-BP1 and by activating AKT by mTORC2 $(5,20)$. The present study demonstrated the antiproliferative effect of RAD001, PP242 and NVP-BEZ235 on CRC cells using MTT and proliferation assays. This data revealed that NVP-BEZ235 $(1 \mu \mathrm{M})$ markedly reduced the viability of all cell lines. Consistent with the antiproliferative effects of NVP-BEZ235, current literature reveals that NVP-BEZ235 inhibits cell proliferation in different cancer cell lines $(21,22)$. However, the present study revealed that in SW480 and CSC480 cells, RAD001 had a slightly better efficacy than PP242 and NVP-BEZ235. Furthermore, when the cells were exposed to the different drugs under hypoxia, significant resistance was observed in all cell lines except for HCT116 which was significantly sensitive to PP242 and NVP-BEZ235. HCT116 is wild-type for APC and has an heterozygous mutation in $\beta$-catenin which acts in a dominant negative fashion increasing $\beta$-catenin/TCF-4-mediated transcriptional activity (23). Newton et al (2010) observed that downregulation of APC is important for survival under hypoxia (24). APC is negative regulator of $\beta$-catenin and mutations in APC are commonly associated with increased $\beta$-catenin activity (23). Recent evidence suggests crosstalk between hypoxia and the upregulation of $\beta$-catenin activity leading to increased cell proliferation migration and invasion (25). Therefore, the wild-type APC gene could be contributing to the apparent sensitivity of HCT116 to PP242 and NVP-BEZ235 through the suppression of $\beta$-catenin activity.

To determine the effects of the mTOR inhibitors on the induction of apoptosis via the mitochondrial pathway, the mitochondrial potential of CRC cells was analysed in the presence of the drugs. Induction of apoptosis by the mitochondria is due to the release of cytochrome $c$ in response to loss of mitochondrial potential (26). Our data revealed that the mTOR inhibitors did not demonstrate significant disruption of the mitochondrial potential, with the exception of PP242 having a slight effect on SW620 cells. The data indicated that NVP-BEZ235 and RAD001 did not induce apoptosis via the mitochondrial pathway. In agreement with our results, induction of apoptosis in breast cancer cells by NVP-BEZ235 was found to be independent of mitochondrial-induced caspases, but rather induced via the extrinsic caspase-7 pathway $(27,28)$. Furthermore, NVP-BEZ235 was demonstrated not to induce the activation of Bax, a key initiator of mitochondrial-induced apoptosis in the human neuroblastoma cell line SH-EP (29).

To ascertain the effect of NVP-BEZ235 on the mTOR pathway, we conducted a phospho-antibody microarray analysis of the key proteins in the mTOR pathway using SW620 cells. Using an mTOR-specific phospho array, changes in the expression levels of phosphoproteins were identified as follows: Downstream target of mTORC1, 4E-BP1 at both sites Thr37 and Thr46 and upstream effector of mTORC2, AKT at site Ser473.

Comparing between 4E-BP1 and S6K as indicators of the functional state of $\mathrm{mTOR}$, a recent study revealed that 
the phosphorylation status of $4 \mathrm{E}-\mathrm{BP} 1$ is a better biomarker than S6K to assess the efficacy ATP-competitive inhibitors (30). mTORC1 regulates protein synthesis through the phosphorylation and inactivation of 4E-BP1 (15). When 4E-BP1 is not phosphorylated (inactive), it binds tightly to eukaryotic translation initiation factor 4E (eIF4E). However, when phosphorylated by mTORC1, 4E-BP1 is released from eIF4E resulting in inhibition of cap-dependent translation and recruitment of the translation initiation factor eIF4G and other cap-binding proteins (3). A previous study reported that RAD001 partially inhibited 4E-BP1 Thr37/46 phosphorylation (31). Our data revealed no effect of RAD001 on 4EBP1 Thr37/46 effector pathway downstream of mTORC1 in all colorectal cell lines. Furthermore, when PP242 (1,000 nM) was used to treat the cells it was found to have a weak inhibitory effect on 4E-BP1 Thr37/46. However, at the same dose NVP-BEZ235 effectively inhibited 4E-BP1 phosphorylation (at Thr37/46). Conversely, we found that in SW480 cells, mTORC1 inhibition by NVP-BEZ235 did not affect the phosphorylation of 4E-BP1, leading us to suggest that alternative mechanisms could also account for the increased 4E-BP1.

The mTORC 2 complex phosphorylates protein kinase AKT at a serine residue Ser473 (5). It has been previously reported that NVP-BEZ235 did not inhibit p-AKT in some cancer cell lines at low concentrations (1-10 $\mathrm{nM})$ and that the inhibitory effect lasted for $48 \mathrm{~h}$ at higher concentrations (21). On the basis of this, we chose to treat the cancer cells with mTOR inhibitors at the concentrations of $1,000 \mathrm{nM}$ for $2 \mathrm{~h}$ to conduct our experiments. We observed that RAD001 increased AKT phosphorylation in SW620 cells. This observation is consistent with previous studies $(32,33)$. In the present study however, PP242 and NVP-BEZ235 decreased p-AKT levels in all cell lines. This is in agreement with previous studies, in which both inhibitors were shown to decrease AKT phosphorylation at high doses $(21,34)$.

In conclusion, our preclinical findings demonstrated that the new generation, ATP-competitive and dual mTOR/PI3K inhibitors of mTOR, are potent in inhibiting CRC cells in vitro. In respect to apoptosis, however, NVP-BEZ235 did not affect the mitochondrial potential. Collectively, these results demonstrated the potential of the new generation of dual mTOR inhibitors in the treatment of CRC. However, further mechanistic, in vivo studies and clinical studies are required to ascertain their usefulness.

\section{Acknowledgements}

We would like to thank the other members of the Wei Laboratory for their support and helpful comments.

\section{Funding}

The present study was supported by a PhD scholarship awarded to Naif Alqurashi by Imam Abdulrahman Bin Faisal University.

\section{Availability of data and materials}

The datasets used during the present study are available from the corresponding author upon reasonable request.

\section{Authors' contributions}

NA conceived, designed and performed experiments. SMH and FA performed experiments and contributed to the writing of the manuscript. SMH, SI and MW reviewed and edited the manuscript. All authors read and approved the manuscript and agree to be accountable for all aspects of the research in ensuring that the accuracy or integrity of any part of the work are appropriately investigated and resolved.

\section{Ethics approval and consent to participate}

Not applicable.

\section{Consent for publication}

Not applicable.

\section{Competing interests}

The authors state that they have no competing interests.

\section{References}

1. Ferlay J, Soerjomataram I, Ervik M, Dikshit R, Eser S, Mathers C, Rebelo M, Parkin DM, Forman D and Bray F: Cancer incidence and mortality worldwide: sources, methods and major patterns in GLOBOCAN 2012. Int J Cancer 136: E359-E386: 2015.

2. Laplante M and Sabatini DM: mTOR signaling in growth control and disease. Cell 149: 274-293, 2012.

3. Zoncu R, Efeyan A and Sabatini DM: mTOR: From growth signal integration to cancer, diabetes and ageing. Nat Rev Mol Cell Biol 12: 21-35, 2011.

4. Laplante M and Sabatini DM: mTOR signaling at a glance. J Cell Sci 122: 3589-3594, 2009.

5. Sarbassov DD, Guertin DA, Ali SM and Sabatini DM Phosphorylation and regulation of Akt/PKB by the rictor-mTOR complex. Science 307: 1098-1101, 2005.

6. Choo AY, Yoon SO, Kim SG, Roux PP and Blenis J: Rapamycin differentially inhibits S6Ks and 4E-BP1 to mediate cell-type-specific repression of mRNA translation. Proc Natl Acad Sci USA 105: 17414-17419, 2008.

7. Guertin DA and Sabatini DM: The pharmacology of mTOR inhibition. Sci Signal 2: pe24, 2009.

8. Zhang YJ, Duan Y and Zheng XF: Targeting the mTOR kinase domain: The second generation of mTOR inhibitors. Drug Discov Today 16: 325-331, 2011.

9. Yu K, Toral-Barza L, Shi C, Zhang WG, Lucas J, Shor B, Kim J, Verheijen J, Curran K, Malwitz DJ, et al: Biochemical, cellular, and in vivo activity of novel ATP-competitive and selective inhibitors of the mammalian target of rapamycin. Cancer Res 69: 6232-6240, 2009.

10. Zaytseva YY, Valentino JD, Gulhati P and Evers BM: mTOR inhibitors in cancer therapy. Cancer Lett 319: 1-7, 2012.

11. Zhang YJ, Dai Q, Sun DF, Xiong H, Tian XQ, Gao FH, Xu MH, Chen GQ, Han ZG and Fang JY: mTOR signaling pathway is a target for the treatment of colorectal cancer. Ann Surg Oncol 16: 2617-2628, 2009

12. Gulhati P, Cai Q, Li J, Liu J, Rychahou PG, Qiu S, Lee EY, Silva SR, Bowen KA, Gao T and Evers BM: Targeted inhibition of mammalian target of rapamycin signaling inhibits tumorigenesis of colorectal cancer. Clin Cancer Res 15: 7207-7216, 2009.

13. Qian Y: Tumorigenic CancerStemCells, Methods of Isolating and Using the Same. Google Patents, US20110206735A1, 2011.

14. Bedner E, Li X, Gorczyca W, Melamed MR and Darzynkiewicz Z: Analysis of apoptosis by laser scanning cytometry. Cytometry 35: 181-195, 1999.

15. Ma XM and Blenis J: Molecular mechanisms of mTOR-mediated translational control. Nat Rev Mol Cell Biol 10: 307-318, 2009.

16. O'Reilly KE, Rojo F, She QB, Solit D, Mills GB, Smith D, Lane H, Hofmann F, Hicklin DJ, Ludwig DL, et al: mTOR inhibition induces upstream receptor tyrosine kinase signaling and activates Akt. Cancer Res 66: 1500-1508, 2006. 
17. Huang S, Bjornsti MA and Houghton PJ: Rapamycins: Mechanism of action and cellular resistance. Cancer Biol Ther 2: 222-232, 2003

18. Gossage L and Eisen T: Targeting multiple kinase pathways: A change in paradigm. Clin Cancer Res 16: 1973-1978, 2010.

19. Alqurashi N, Hashimi SM and Wei MQ: Chemical inhibitors and microRNAs (miRNA) targeting the mammalian target of rapamycin (mTOR) pathway: Potential for novel anticancer therapeutics. Int J Mol Sci 14: 3874-3900, 2013.

20. Dowling RJ, Topisirovic I, Alain T, Bidinosti M, Fonseca BD, Petroulakis E, Wang X, Larsson O, Selvaraj A, Liu Y, et al: mTORC1-mediated cell proliferation, but not cell growth, controlled by the 4E-BPs. Science 328: 1172-1176, 2010.

21. Serra V, Markman B, Scaltriti M, Eichhorn PJ, Valero V, Guzman M, Botero ML, Llonch E, Atzori F, Di Cosimo S, et al: NVP-BEZ235, a dual PI3K/mTOR inhibitor, prevents PI3K signaling and inhibits the growth of cancer cells with activating PI3K mutations. Cancer Res 68: 8022-8030, 2008.

22. Roper J, Richardson MP, Wang WV, Richard LG, Chen W, Coffee EM, Sinnamon MJ, Lee L, Chen PC, Bronson RT, et al: The dual PI3K/mTOR inhibitor NVP-BEZ235 induces tumor regression in a genetically engineered mouse model of PIK3CA wild-type colorectal cancer. PLoS One 6: e25132, 2011.

23. Ilyas M, Tomlinson IP, Rowan A, Pignatelli M and Bodmer WF: Beta-catenin mutations in cell lines established from human colorectal cancers. Proc Natl Acad Sci USA 94: 10330-10334, 1997.

24. Newton IP, Kenneth NS, Appleton PL, Näthke I and Rocha S: Adenomatous polyposis coli and hypoxia-inducible factor-1\{alpha\} have an antagonistic connection. Mol Biol Cell 21: 3630-3638, 2010

25. Xu W, Zhou W, Cheng M, Wang J, Liu Z, He S, Luo X, Huang W, Chen T, Yan W and Xiao J: Hypoxia activates Wnt/ $\beta$-catenin signaling by regulating the expression of BCL9 in human hepatocellular carcinoma. Sci Rep 7: 40446, 2017.

26. Perry SW, Norman JP, Barbieri J, Brown EB and Gelbard HA: Mitochondrial membrane potential probes and the proton gradient: A practical usage guide. Biotechniques 50: 98-115, 2011.
27. Brachmann SM, Hofmann I, Schnell C, Fritsch C, Wee S, Lane H, Wang S, Garcia-Echeverria C and Maira SM: Specific apoptosis induction by the dual PI3K/mTor inhibitor NVP-BEZ235 in HER 2 amplified and PIK3CA mutant breast cancer cells. Proc Natl Acad Sci USA 106: 22299-22304, 2009.

28. Cuda CM, Pope RM and Perlman H: The inflammatory role of phagocyte apoptotic pathways in rheumatic diseases. Nat Rev Rheumatol 12: 543-558, 2016.

29. Seitz C, Hugle M, Cristofanon S, Tchoghandjian A and Fulda S: The dual PI3K/mTOR inhibitor NVP-BEZ235 and chloroquine synergize to trigger apoptosis via mitochondrial-lysosomal cross-talk. Int J Cancer 132: 2682-2693, 2013.

30. Ducker GS, Atreya CE, Simko JP, Hom YK, Matli MR, Benes CH, Hann B, Nakakura EK, Bergsland EK, Donner DB, et al: Incomplete inhibition of phosphorylation of $4 \mathrm{E}-\mathrm{BP} 1$ as a mechanism of primary resistance to ATP-competitive mTOR inhibitors. Oncogene 33: 1590-1600, 2014.

31. Thoreen CC, Kang SA, Chang JW, Liu Q, Zhang J, Gao Y, Reichling LJ, Sim T, Sabatini DM and Gray NS: An ATP-competitive mammalian target of rapamycin inhibitor reveals rapamycin-resistant functions of mTORC1. J Biol Chem 284: 8023-8032, 2009.

32. Wang X, Yue P, Kim YA, Fu H, Khuri FR and Sun SY: Enhancing mammalian target of rapamycin (mTOR)-targeted cancer therapy by preventing mTOR/raptor inhibition-initiated, mTOR/rictor-independent Akt activation. Cancer Res 68: 7409-7418, 2008.

33. Xu CX, Li Y, Yue P, Owonikoko TK, Ramalingam SS, Khuri FR and Sun SY: The combination of RAD001 and NVP-BEZ235 exerts synergistic anticancer activity against non-small cell lung cancer in vitro and in vivo. PLoS One 6: e20899, 2011

34. Blaser B, Waselle L, Dormond-Meuwly A, Dufour M, Roulin D, Demartines $\mathrm{N}$ and Dormond O: Antitumor activities of ATP-competitive inhibitors of mTOR in colon cancer cells. BMC Cancer 12: 86, 2012. 